\title{
Asymptotic analysis of multi-branch two-way amplify-and-forward relaying in Nakagami- $m$ fading with arbitrary fading parameter
}

\author{
Jing YANG ${ }^{*}$, Pingzhi FAN \\ Institute of Mobile Communications, Southwest Jiaotong University, Chengdu 610031, China
}

\begin{abstract}
Two-way relay networks have received lots of attention, thanks to its ability to overcome the loss in the spectral efficiency due to half-duplex transmission. Asymptotic performance analysis can provide valuable insights into practical system designs. However, this is a gap in two-way relay network. In this paper, the asymptotic performance is studied for multi-branch dual-hop two-way amplify-and-forward (AF) relaying networks in independently but not necessarily identically distributed (i.n.i.d.) Nakagami- $m$ fading channels, with arbitrary $m \geq 5$. The approximate probability density function (PDF) of the instantaneous dual-hop link power at high SNR region is derived. Then we present the asymptotic outage probability expression, and analyze the diversity order and coding gain. Simulations are performed to verify the tightness of the presented analysis at medium and high SNR regions.
\end{abstract}

Key words: two-way relay network; amplify-and-forward; outage probability; average bit error probability; Nakagami$m$ fading

(C) 2012 JMT. All rights reserved.

\section{Introduction}

$\mathrm{R}$ ecently, two-way relay networks have received lots of attention, where two source terminals exchange information with each other through multiple intermediate relay terminals. By allowing concurrent transmission of two information flows, two-way networks can mitigate the spectral loss caused by the halfduplex mode of relays [1]. In [1], Rankov and Wittneben introduced the two-way relaying protocols, known as two-way amplify-and-forward (AF) relaying and twoway decode-and-forward (DF) relaying.

The performance of two-way relaying system has been studied in the past from various aspects. For the special case where Rayleigh fading is assumed, several analytical results are derived, including the exact outage probability expression [2], bounds for average sum-rate and pairwise error probability (PEP) [3], the exact closed-form expressions for average symbol error rate (SER) and average sum-rate [4], and the closed-form expressions for overall outage probability and SER [5]. Considering mixed Rayleigh and Rician fading,

Received Oct. 21, 2012; revision accepted Nov. 1, 2012

*Corresponding author. E-mail: jingyang905@163.com (J.

YANG)

(C) 2012 JMT. All rights reserved

doi: 10.3969/j.issn.2095-087X.2012.04.009
Refs. [6-7] studied the asymmetric fading conditions. Refs. [8-11] studied relay selection from various points for two-way relay networks. Amarasuriya et.al. [12] studied the performance of zero-forcing for two-way MIMO AF relay networks. Refs. [13-14] studied the impact of outdated channel state information on the performance of two-way relay networks. Later, Ilhan et.al. [15] studied the performance in cascaded Nakagami- $m$ fading. In Nakagami-m fading environment with integer and integer plus one-half values of fading parameter $m$, Yang et al. [16] presented the exact closed form expressions for outage probability, average SER, and average sum-rate. However, in practical scenarios, the parameter $m$ often adopts arbitrary values [17], which exclude the generality of Ref. [16].

This paper, with arbitrary $m \geq 0.5$, analyzes the large SNR performance for multi-branch dual-hop two-way AF relaying system in terms of outage probability. We first study the dual-hop channel instantaneous output signalto-noise ratio (SNR) and provide its approximate PDF at high SNR region, and then extend it to multi-branch systems. Further, asymptotic expressions of outage probability are obtained. Our results show that the diversity and coding gains are solely determined by the more severely faded link. The analytical results are verified by simulation results, indicating that the derived results are tight particularly at medium and high SNR regions. 


\section{System and channel model}

A two-way AF relay network (depicted as Fig. 1) is considered, where two terminals, $T_{1}$ and $T_{2}$, are required to exchange information with each other through multiple intermediate relay terminals, labeled as $R_{1}, R_{2}, \cdots, R_{N}$. Every terminal has only one antenna and is half-duplex. Communication takes place over $N+1$ time slots. In the first time slot, $T_{1}$ and $T_{2}$ simultaneously send their information message to $\{R\}_{i=1}^{N}$. After receiving the signal, $R_{i}$ normalizes the received signal based on its transmit power constraint and broadcasts it to $T_{1}$ and $T_{2}$ in the $(i+1)$ th time slot. Finally, the selfinterference are fully eliminated at $T_{1}$ and $T_{2}$, respectively. As in Ref. [6], we assume equal power (denoted by $P_{b}$ ) at $T_{1}, T_{2}$, and $\{R\}_{i=1}^{N}$ and equal variance (denoted by $N_{0}$ ) for the additive white Gaussian noise (AWGN) at all terminals. Besides channels are reciprocal, so the channel gains of $T_{u} \rightarrow R$ and $R \rightarrow T_{u}(u=1,2)$ links are identical [1].

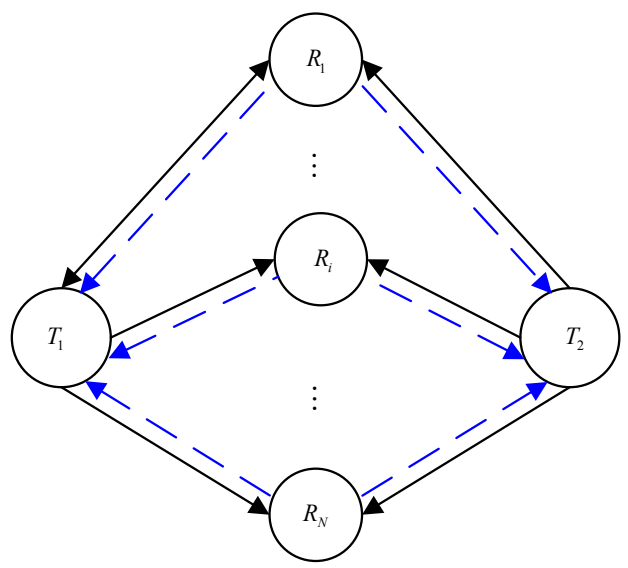

Fig. 1 System model

The instantaneous output SNR of any relay link $T_{u} \rightarrow R \rightarrow T_{v}(u, v=1,2)$ is $[1,3-4]$

$$
\gamma_{i}=\bar{\gamma} \frac{h_{i 1} h_{i 2}}{2 h_{i 2}+h_{i 1}+1 / \bar{\gamma}},
$$

where $h_{i 1}$ and $h_{i 2}(i=1,2, \cdots, N)$ are the powers of instantaneous channel envelops for $T_{u} \rightarrow R_{i}$ and $R_{i} \rightarrow T_{v}$ links, respectively. Note that $\bar{\gamma}=P_{b} / N_{0}$ is the average transmit bit SNR and

$$
h_{i 1} \sim G\left(m_{i 1}, \frac{\Omega_{i 1}}{m_{i 1}}\right), \quad h_{i 2} \sim G\left(m_{i 2}, \frac{\Omega_{i 2}}{m_{i 2}}\right),
$$

where $G(\cdot, \cdot)$ is incomplete Gamma function; $\Omega_{i 1}$ and $\Omega_{i 2}$ are the respective average fading powers.

Assuming the maximum ratio combining (MRC) is performed at $T_{v}$, the total received signal-to-noise ratio (SNR) can be written as

$$
\gamma=\sum_{i=1}^{N} \gamma_{i}
$$

\section{Asymptotic performance analysis of two- way AF relaying network}

In this section, we present the closed-form expression for the asymptotic average bit error probability (ABEP) and out-age probability.

The results can be examined in terms of the diversity and coding gain, which are two important measures of the network. Firstly, we present the approximations of $\gamma_{i}$ 's distribution, as the SNR of the system is sufficiently high.

\subsection{Distribution of the received SNR}

Proposition 1 Consider the two independent Gamma distributed random $h_{i 1}$ and $h_{i 2}$. Define $m_{i}=\min \left(m_{i 1}, m_{i 2}\right)$ and let $z>0$. The distribution of $\gamma_{i}$ follows the property of

$$
\lim _{\bar{\gamma} \rightarrow \infty} \frac{P\left[\gamma_{i}<z\right]}{(z / \bar{\gamma})^{m_{i}}}=\frac{\beta_{i}}{m_{i}},
$$

where

$$
\beta_{i}= \begin{cases}\frac{\left(\frac{2 m_{i 1}}{\Omega_{i 1}}\right)^{m_{i 1}}}{\Gamma\left(m_{i 1}\right)}, & m_{i 1}<m_{i 2}, \\ \frac{\left(\frac{m_{i 2}}{\Omega_{i 2}}\right)^{m_{i 2}}}{\Gamma\left(m_{i 2}\right)}, & m_{i 2}<m_{i 1}, \\ \frac{\left(\frac{2 m_{i 1}}{\Omega_{i 1}}\right)^{m_{i 1}}}{\Gamma\left(m_{i 1}\right)}+\frac{\left(\frac{m_{i 2}}{\Omega_{i 2}}\right)^{m_{i 2}}}{\Gamma\left(m_{i 2}\right)}, & m_{i 2}=m_{i 1},\end{cases}
$$

and $\Gamma(\cdot)$ is the Gamma function.

Proof: We begin with the lower bound, i.e.,

$$
\lim _{\bar{\gamma} \rightarrow \infty} \frac{P\left[\gamma_{i}<z\right]}{\left(\frac{z}{\bar{\gamma}}\right)^{m_{i}}} \geq \frac{\beta_{i}}{m_{i}} .
$$


Observe that $\gamma_{i} \leq \bar{\gamma} \min \left(\frac{h_{i 1}}{2}, h_{i 2}\right)$. Then, we have

$$
\begin{aligned}
& P\left[\gamma_{i}<z\right]=P\left[\frac{\frac{h_{i 1}}{2} h_{i 2}}{\frac{h_{i 1}}{2}+h_{i 2}+\frac{1}{2 \bar{\gamma}}}<\frac{z}{\bar{\gamma}}\right] \geq \\
& P\left[\min \left(\frac{h_{i 1}}{2}, h_{i 2}\right)<\frac{z}{\bar{\gamma}}\right]= \\
& 1-P\left[\frac{h_{i 1}}{2}>\frac{z}{\bar{\gamma}}\right] P\left[h_{i 2}>\frac{z}{\bar{\gamma}}\right]= \\
& 1-\frac{\left(\frac{2 m_{i 1}}{\Omega_{i 1}}\right)^{m_{i 1}}}{\Gamma\left(m_{i 1}\right)}+\frac{\left(\frac{m_{i 2}}{\Omega_{i 2}}\right)^{m_{i 2}}}{\Gamma\left(m_{i 2}\right)} .
\end{aligned}
$$

Note that one of the series representations of the upper incomplete Gamma function is as follows [18]:

$$
\Gamma(a, x)=\int_{x}^{\infty} t^{a-1} \exp (-t) \mathrm{d} t=\Gamma(a)-\sum_{m=0}^{\infty} \frac{(-1)^{n} x^{a+n}}{n !},
$$

and (5) can be expanded as

$$
\begin{aligned}
& P\left[\gamma_{i}<z\right] \overbrace{\frac{1}{\Gamma\left(m_{i 1}\right)} \sum_{n=0}^{\infty} \frac{(-1)^{n}\left(\frac{2 m_{i 1}}{\Omega_{i 1}} \frac{z}{\bar{\gamma}}\right)^{m_{i 1}+n}}{n !\left(m_{i 1}+n\right)}}^{J_{1}}+ \\
& \frac{1}{\Gamma\left(m_{i 2}\right)} \overbrace{l=0}^{J_{2}} \frac{(-1)^{l}\left(\frac{m_{i 2}}{\Omega_{i 2}} \frac{z}{\bar{\gamma}}\right)^{m_{i 2}+l}}{l !\left(m_{i 2}+l\right)}- \\
& \frac{1}{\Gamma\left(m_{i 1}\right)} \frac{1}{\Gamma\left(m_{i 2}\right)} J_{1} J_{2} .
\end{aligned}
$$

Applying the limit operation when $\bar{\gamma} \rightarrow \infty$, (6) consists of three infinitesimals with orders of $m_{i 1}, m_{i 2}$ and $m_{i 1}+m_{i 2}$. Therefore, the right side of (6) is an infinitesimal of the $m_{i}$ th order:

$$
\lim _{\bar{\gamma} \rightarrow \infty} \frac{P\left[\gamma_{i}<z\right]}{\left(\frac{z}{\bar{\gamma}}\right)^{m_{i}}} \geq \frac{\beta_{i}}{m_{i}},
$$

where $m_{t}$ and $\beta_{i}$ are defined in (3).

In the other direction, i.e., the upper bound, let $l>1$ and $k>l$ be two fixed constants. Then

$$
P\left[\gamma_{i}<z\right]=P\left[\frac{\frac{h_{i 1}}{2} h_{i 2}}{\frac{h_{i 1}}{2}+h_{i 2}+\frac{1}{2 \bar{\gamma}}}<z / \bar{\gamma}\right]=
$$

$$
\begin{aligned}
& P\left[h_{i 1}\left(h_{i 2}-\frac{z}{\bar{\gamma}} \leq \frac{2 z}{\bar{\gamma}}\left(h_{i 2}+\frac{1}{2 \bar{\gamma}}\right)\right)\right] \leq \\
& \int_{0}^{l \frac{z}{\bar{\gamma}}} f_{h_{i 2}} \mathrm{~d} h_{i 2}+\int_{k \frac{z}{\bar{\gamma}}}^{\infty} P\left[h_{i 1}<\frac{\frac{2 z}{\bar{\gamma}}\left(h_{i 2}+\frac{1}{2 \bar{\gamma}}\right)}{h_{i 2}-\frac{z}{\bar{\gamma}}}\right] f_{h_{i 2}} \mathrm{~d} h_{i 2}+ \\
& \int_{l \frac{z}{\bar{\gamma}}}^{k \frac{z}{\bar{\gamma}}} P\left[h_{i 1}<\frac{\frac{2 z}{\bar{\gamma}}\left(h_{i 2}+\frac{1}{2 \bar{\gamma}}\right)}{h_{i 2}-\frac{z}{\bar{\gamma}}}\right] f_{h_{i 2}} \mathrm{~d} h_{i 2}=J_{3}+J_{4}+J_{5} .
\end{aligned}
$$

For $J_{3}$,

$$
\begin{gathered}
\lim _{\bar{\gamma} \rightarrow \infty} \frac{J_{3}}{\left(\frac{z}{\bar{\gamma}}\right)^{m_{i 2}}}=\lim _{\bar{\gamma} \rightarrow \infty} \frac{1-\Gamma\left(m_{i 2}, \frac{m_{i 2}}{\Omega_{i 2}} \frac{l z}{\bar{\gamma}}\right)}{\Gamma\left(m_{i 2}\right)\left(\frac{z}{\bar{\gamma}}\right)^{m_{i 2}}}= \\
\frac{1}{\Gamma\left(m_{i 2}\right)}\left(l \frac{m_{i 2}}{\Omega_{i 2}}\right)^{m_{i 2}} .
\end{gathered}
$$

For $J_{4}$ the fact that

$$
P\left[h_{i 1}<\frac{2 z}{\bar{\gamma}}\left(h_{i 2}+\frac{1}{2 \bar{\gamma}}\right) /\left(h_{i 2}-\frac{z}{\bar{\gamma}}\right)\right]
$$

is non-increasing in $h_{i 2}$ leads to

$$
\begin{gathered}
J_{4} \leq P\left[h_{i 1}<\frac{\frac{2 z}{\bar{\gamma}}\left(k \frac{z}{\bar{\gamma}}+\frac{1}{2 \bar{\gamma}}\right)}{k \frac{z}{\bar{\gamma}}-\frac{z}{\bar{\gamma}}}\right]= \\
P\left[h_{i 1}<\frac{2\left(k \frac{z}{\bar{\gamma}}+\frac{1}{2 \bar{\gamma}}\right)}{k-1}\right]=P\left\{h_{i 1}<c\right\} .
\end{gathered}
$$

And hence,

$$
\begin{gathered}
\lim _{\gamma \rightarrow \infty} \frac{J_{4}}{\left(\frac{z}{\bar{\gamma}}\right)^{m_{i 1}}} \leq \lim _{\gamma \rightarrow \infty} \frac{1-\Gamma\left(m_{i 1}, \frac{c m_{i 1}}{\Omega_{i 1}}\right)}{\Gamma\left(m_{i 1}\right)\left(\frac{z}{\bar{\gamma}}\right)^{m_{i 1}}}= \\
\frac{1}{m_{i 1} \Gamma\left(m_{i 1}\right)}\left(\frac{2 m_{i 1}}{\Omega_{i 1}} \frac{k}{k-1}\right)^{m_{i 1}} .
\end{gathered}
$$

Moreover, by changing variable $y=h_{i 2} /(z / \bar{\gamma})$ and introducing exact PDF of $h_{i 2}, J_{5}$ can be rewritten as 


$$
\begin{aligned}
& J_{5}=\int_{u}^{v} \Gamma\left(m_{i 1}, \frac{2\left(y \frac{z}{\bar{\gamma}}+\frac{1}{2 \bar{\gamma}}\right)}{y-1}\right) \times \\
& \frac{1}{m_{i 2}}\left(\frac{m_{i 2}}{\Omega_{i 2}}\right)^{m_{i 2}} y^{m_{i 2}-1}\left(\frac{z}{\bar{\gamma}}\right)^{m_{i 2}} \exp \left(-\frac{m_{i 2}}{\Omega_{i 2}} \frac{z}{\bar{\gamma}} y\right) \mathrm{d} y= \\
& \frac{1}{\Gamma\left(m_{i 1}\right) \Gamma\left(m_{i 2}\right)}\left(\frac{m_{i 2}}{\Omega_{i 2}}\right)^{m_{i 2}} \sum_{n=0}^{\infty} \frac{(-1)^{n}}{n !\left(m_{i 1}+n\right)} \times \\
& \left(\frac{z}{\bar{\gamma}}\right)^{m_{i 1}+m_{i 2}+n}\left(\frac{m_{i 1}}{\Omega_{i 1}}\right)^{m_{i 1}+n} \times \\
& \int_{l}^{k}(\frac{\left.2\left(y+\frac{1}{2 z}\right)\right)_{J_{6}}^{m_{i 1}+n}}{y-1} \underbrace{y^{m_{i 2}-1} \exp \left(-\frac{m_{i 2}}{\Omega_{i 2}} \frac{z}{\bar{\gamma}} y\right) \mathrm{d} y,}
\end{aligned}
$$

$J_{6}$ remains finite for any $v>u>1$ as $\bar{\gamma} \rightarrow \infty$, which makes

$$
\lim _{\bar{\gamma} \rightarrow \infty} \frac{J_{5}}{\left(\frac{z}{\bar{\gamma}}\right)^{m_{i}}} \leq \frac{\beta_{i}}{m_{i}} .
$$

Noticing that (10), (11) and (12) are valid for arbitrary constants $k>l>1$, here we choose arbitrary $k$ and $l$ close to 1 . Hence,

$$
\lim _{\bar{\gamma} \rightarrow \infty} \frac{P\left[\gamma_{i}<z\right]}{(z / \bar{\gamma})^{m_{i}}} \leq \frac{\beta_{i}}{m_{i}} .
$$

Combining (7) and (14), Proposition 1 is achieved.

As the exact expression of the PDF of $\gamma_{i}$ is complicated, a more general and simple presentation is available in high SNR scenarios:

$$
f_{\gamma_{i}}\left(\gamma_{i}\right)=\beta_{i} \gamma_{i}^{m_{i}-1}+o\left(\gamma_{i}^{m_{i}-1+\varepsilon}\right), \varepsilon>0 .
$$

where $o(\cdot)$ denotes the higher order terms.

Proposition 2 Let $X=\sum_{i=1}^{N} \gamma_{i}$, where $\gamma_{i}$ satisfies (3), the distribution of $X$ follows the property of

$$
\lim _{\bar{\gamma} \rightarrow \infty} \frac{p\left[\gamma_{i}<z\right]}{(z / \bar{\gamma})^{m_{\mathrm{AF}}}}=\frac{\prod_{i=1}^{N} \Gamma\left(m_{i}\right) \beta_{i}}{\Gamma\left(m_{\mathrm{AF}}+1\right)},
$$

where $m_{A F}=\sum_{i=1}^{N} m_{i}$.
Proof: Begin with Proposition 2 with $N=2$, i.e., $X=\gamma_{1}+\gamma_{2} ;$ and

$$
\begin{gathered}
P[X<z]=\int_{0}^{z} P\left[\gamma_{1}<z-\gamma_{2}\right] f_{\gamma_{2}} \mathrm{~d} \gamma_{2}= \\
\int_{0}^{z}\left[\frac{\beta_{1}}{m_{1}}\left(\frac{z-\gamma_{2}}{\bar{\gamma}}\right)^{m_{1}}+H . O . T\right] \times \\
{\left[\beta_{2} \bar{\gamma}^{m_{2}} \gamma_{2}^{m_{2}-1}+\text { H.O.T }\right] \mathrm{d} \gamma_{2} .}
\end{gathered}
$$

Keeping $y=\gamma_{2} / z$ variable, and ignoring the higher order terms H.O.T, we have

$$
\begin{aligned}
& \lim _{\gamma \rightarrow \infty} \frac{P[X<z]}{(z / \bar{\gamma})^{m_{1}+m_{2}}}=\lim _{\gamma \rightarrow \infty} \frac{1}{(z / \bar{\gamma})^{m_{1}+m_{2}}} \times \\
& \int_{0}^{z} \frac{\beta_{1}}{m_{1}}\left(\frac{z-\gamma_{2}}{\bar{\gamma}}\right)^{m_{1}} \times \beta_{2} \bar{\gamma}^{-m_{2}} \gamma_{2}^{m_{2}-1} \mathrm{~d} \gamma_{2}= \\
& \frac{\beta_{1} \beta_{2}}{m_{1}} \int_{0}^{1}(1-y)^{m_{1}} y^{m_{2}-1} \mathrm{~d} y= \\
& \frac{\beta_{1} \beta_{2}}{m_{1}} B\left(m_{1}+1, m_{2}\right),
\end{aligned}
$$

where $B(\cdot, \cdot) \quad[18]$ is the Beta function. Using $B(x, y)=\Gamma(x)=\Gamma(y) / \Gamma(x+y) \quad[18] \quad$ and $\quad \Gamma\left(m_{1}+1\right)=$ $\Gamma\left(m_{1}+1\right)=m_{1} \Gamma\left(m_{1}+1\right)$, the right ride of (18) would be $\beta_{1} \beta_{2} \Gamma\left(m_{1}\right) \Gamma\left(m_{2}\right) / \Gamma\left(m_{1}\right)+\Gamma\left(m_{2}+1\right)$. Extending the results to multiple variable cases, one can get (16). The proof is completed.

\section{2. $A B E P$}

According to [19], the asymptotic ABEP and outage probability can be derived based on the behavior of $f_{\gamma_{i}}\left(\gamma_{i}\right)$ around the origin.

If the PDF of $\gamma$ can be approximated as $f_{\gamma}(\gamma)=a \gamma^{t}+o\left(\gamma^{t+\varepsilon}\right)\left(\gamma \rightarrow 0^{+}\right.$and $\left.\varepsilon>0\right)$ and instantaneous error probability is given by $P_{E}(k)=Q(\sqrt{k \gamma})$, the asymptotic error probability can be given as follows:

$$
\int_{0}^{\infty} Q(\sqrt{k \gamma}) f_{\gamma}(\gamma) \mathrm{d} \gamma \rightarrow \frac{2^{t} a \Gamma(t+1.5)}{\sqrt{\pi} k^{t+1}(t+1)} .
$$

It is known that for general gray bit-mapped constellations employed in practical systems, the conditional ABEP is a finite sum of weighted $Q$-function

$$
P_{b}(e \mid \gamma)=\sum_{k=1}^{K} A_{k} Q\left(\sqrt{B_{k} \gamma}\right)
$$

where $A_{k}, B_{k}$ and $K$ are modulation type dependent constants [20]. Therefore, the asymptotic presentation of 
ABEP for multi-branch dual-hop two-way AF relaying system is obtained as

$$
P_{b}^{\infty}=\frac{\Phi 2^{m_{\mathrm{AF}}-1} \Gamma\left(m_{\mathrm{AF}}+0.5\right)}{\sqrt{\pi} \Gamma\left(m_{\mathrm{AF}}+1\right) \bar{\gamma}^{m_{\mathrm{AF}}}} \beta_{i} \Gamma\left(m_{i}\right),
$$

where $\Phi=\sum_{k=1}^{K} A_{k} B_{k}^{-m_{\mathrm{AF}}}$.

From (20), the diversity gain $G_{d}$ and the coding gain $G_{c}$ are as follows [19]:

$$
G_{d}=m_{\mathrm{AF}},
$$

and

$$
G_{c}=\left(\frac{\Phi 2^{m_{\mathrm{AF}}-1} \Gamma\left(m_{\mathrm{AF}}+0.5\right)}{\sqrt{\pi} \Gamma\left(m_{\mathrm{AF}}+1\right)} \prod_{i=1}^{N} \beta_{i} \Gamma\left(m_{i}\right)\right)^{-\frac{1}{m_{\mathrm{AF}}}} .
$$

\subsection{Outage probability}

Outage probability $P_{\text {out }}$ is a commonly used performance indicator when communicating over fading channels. It is defined as the probability that the instantaneous SNR $\gamma_{t h}$ falls below a certain threshold $\gamma_{t h}$.

$$
P_{\text {out }}:=P\left(0 \leq \gamma \leq \gamma_{\text {th }}\right)=\int_{0}^{\gamma_{\text {th }}} f_{\gamma}(\gamma) \mathrm{d} \gamma .
$$

Based on (15), for a sufficiently large $\bar{\gamma}$ the outage probability for multi-branch dual-hop two-way AF relaying system is as follows [19]:

$$
P_{\mathrm{out}}^{\infty}=\frac{\prod_{i=1}^{N} \beta_{i} \Gamma\left(m_{i}\right)}{\Gamma\left(m_{\mathrm{AF}}+1\right) \bar{\gamma}^{m_{\mathrm{AF}}}}\left(\frac{\gamma_{\mathrm{th}}}{\bar{\gamma}}\right)^{m_{\mathrm{AF}}}+o\left(\bar{\gamma}^{-(t+1)}\right),
$$

which indicates that the outage diversity $O_{\mathrm{d}}=m_{\mathrm{AF}}$ and the coding gain

$$
O_{\mathrm{c}}=\frac{1}{\gamma_{\mathrm{th}}}\left(\frac{\prod_{i=1}^{N} \beta_{i} \Gamma\left(m_{i}\right)}{\Gamma\left(m_{\mathrm{AF}}+1\right) \bar{\gamma}^{m_{\mathrm{AF}}}}\right)^{-\frac{1}{m_{\mathrm{AF}}}} .
$$

Our results have shown that the resulting diversity and coding gains are solely determined by the more severely faded link.

\section{Numerical and simulation results}

In this section, simulation is provided to demonstrate the validity and usefulness of our analytical expressions derived for ABEP and outage probability. We investigate cooperative systems with up to three relays $\left(m_{i 1}, m_{i 2}\right)=(0.8,2),(1,1),(3,1.7) \quad$ corresponding to the three cases in (4) using quadrature phase shift keying (QPSK) modulation. The choice of non-integer severity parameters of Nakagami- $m$ fading verifies the generality of the presented analysis.

Assume that $\Omega_{i 1}=2$ and $\Omega_{i 2}=1,(i=1,2, \cdots, N)$ and $\gamma_{\text {th }}=3$. Figs. 2 and 3 show the ABEP and outage probability versus $\bar{\gamma}$ at terminal $T_{2}$ with relay sets $\left\{R_{1}\right\},\left\{R_{2}, R_{3}\right\},\left\{R_{1}, R_{2}, R_{3}\right\}$, respectively. It can be seen that the simulation results agree with the asymptotic results very well in the medium and high SNR regions. In addition, the diversity order increases with $N$, which agrees with the analytical expressions in Section 3.

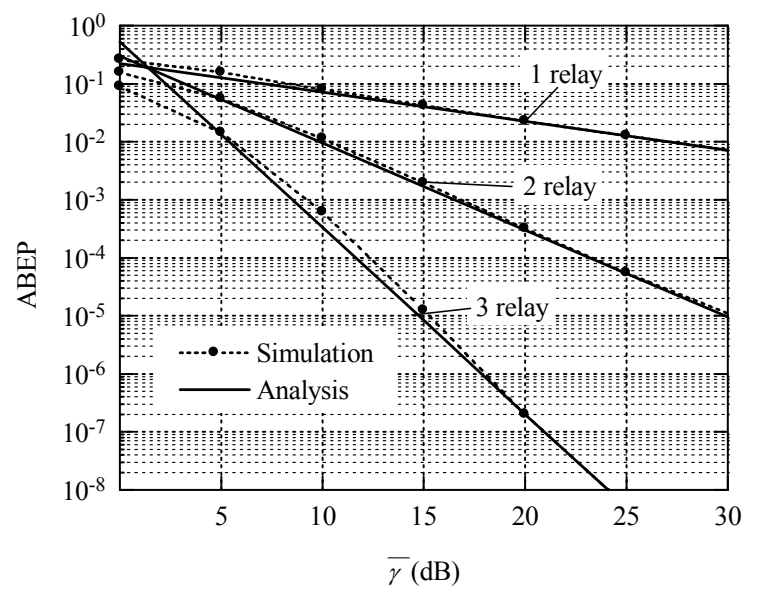

Fig. 2 ABEP of multi-branch dual-hop two-way relaying network with $\left\{R_{1}\right\},\left\{R_{1}, R_{2}\right\}$ and $\left\{R_{1}, R_{2}, R_{3}\right\}$ relays

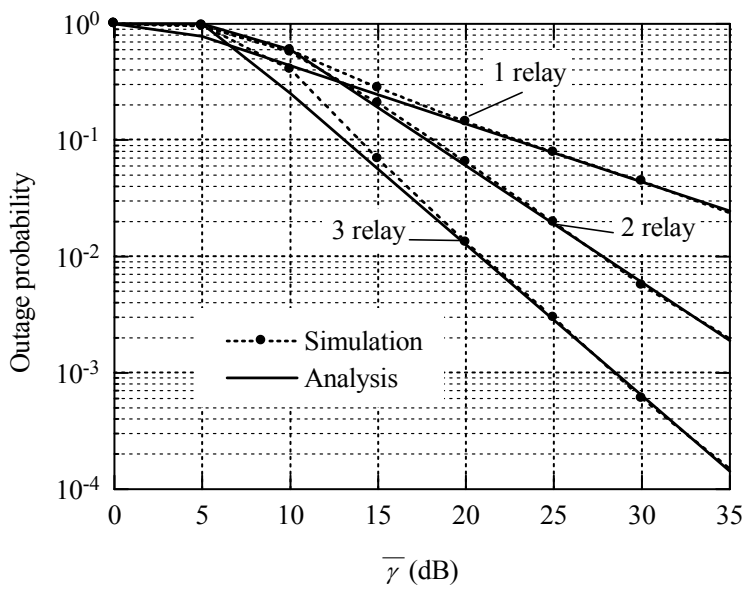

Fig. 3 Outage probability of multi-branch dual-hop two-way relaying network with $\left\{R_{1}\right\},\left\{R_{1}, R_{2}\right\}$ and $\left\{R_{1}, R_{2}, R_{3}\right\}$ relays

\section{Conclusion}

In this paper, the asymptotic performance is studied for multi-branch dual-hop two-way AF relaying net- 
works in i.n.i.d. Nakagami- $m$ fading channels, with arbitrary $m \geq 0.5$. The approximate probability density function (PDF) of the instantaneous dual-hop link power at high SNR region is derived. Utilizing the results, the asymptotic outage probability expressions are presented, from which the diversity order and coding gain are analyzed. The analytical results show that diversity and coding gains are solely determined by the more severely faded link. Simulations are performed to verify the tightness of the presented analysis at medium and high SNR regions.

\section{Acknowledgements}

This work was supported by the Major State Basic Research Development Program of China (973 Program No. 2012CB316100), the National Natural Science Foundation of China (No. 61032002), and the Fundamental Research Funds for the Central Universities (No. 2010XS21).

\section{References}

[1] B. Rankov, A. Wittneben, Spectral efficient protocols for halfduplex fading relay channels, IEEE J. Sel. Areas Commun., 2007, 25(2): 379-389.

[2] Q. Li, S.H. Ting, A. Pandharipande, et al., Adaptive twoway relaying and outage analysis, IEEE Trans. Wireless Commun., 2009, 8(6): 3288-3299.

[3] Y. Han, S.H. Ting, C.K. Ho, et al., Performance bounds for two-way amplify-and-forward relaying, IEEE Trans. Wireless Commun., 2009, 8(1): 432-439.

[4] T.Q. Duong, L.N. Hoang, V.N.Q. Bao, On the performance of two-way amplify-and-forward relay, IEICE Trans. Commun., 2009, 12: 3957-3959.

[5] H. Guo, J. Ge, H. Ding, Symbol error probability of two-way amplify-and-forward relaying, IEEE Commun. Lett., 2011, 15(1): 22-24.

[6] H.A. Suraweera, R.H.Y. Louie, Y.H. Li , et al., Two hop amplify-and-forward transmission in mixed Rayleigh and Rician fading channels, IEEE Commun. Lett., 2009, 13(4): 227-229.

[7] H. Ding, J. Ge, D.B. da Costa, et al., Outage analysis for multiuser two-way relaying in mixed Rayleigh and Rician fading, IEEE Commun.Lett., 2011, 15(4): 410-412.

[8] D. Michalopoulos, A. Lioumpas, G.K. Karagiannidis, et al., Selective cooperative relaying over time-varying channels, IEEE Trans. Commun., 2010, 58(8): 24022412.

[9] Y. Li, R.H.Y. Louie, B. Vucetic, Relay selection with network coding in two-way relay channels, IEEE Trans. Veh. Technol., 2010, 59(9): 4489-4499.

[10] S.I. Chu, Performance of amplify-and-forward cooperative communications with the $N$ th best-relay selection scheme over Nakagami- $m$ fading channels, IEEE Commun. Lett., 2011, 15(2): 172-174.

[11] Y. Li, Q. Yin, W. Xu, et al., On the design of relay selection strategies in regenerative cooperative networks with outdated CSI, IEEE Trans. Wireless Commun., 2011, 10(9): 3086-3097.

[12] G. Amarasuriya, C. Tellambura, M. Ardakani, Performance analysis of zero-forcing for two-way MIMO AF relay networks, IEEE Wireless Commun. Lett., 2012, 1(2): 53-56.

[13] F. Gao, R. Zhang, and Y.-C. Liang, Optimal channel estimation and training design for two-way relay networks, IEEE Trans. Commun., 2009, 57(10): 3024-3033.

[14] M. Torabi, D. Haccoun, Capacity analysis of opportunistic relaying in cooperative systems with outdated channel information, IEEE Commun. Lett., 2010, 14(12): 1137-1139.

[15] H. Ilhan, Performance Analysis of two-way AF relaying systems over cascaded Nakagami- $m$ fading channels, IEEE Signal Processing Lett., 2012, 19(6): 332-335.

[16] J. Yang, P. Fan, T. Duong, et al., Exact performance of two-way AF relaying in Nakagami- $m$ fading environment, IEEE Trans. Wireless Commun., 2011, 10(3): 980987.

[17] R. Lorenzo, R. Juan, C. Narcis, Evaluation of Nakagami fading behaviour based on measurements in urban scenarios, AEU INT. J Electron. and Commun., 2007, 61(2): 135-138.

[18] I.S. Gradshteyn, I.M. Ryzhik, Table of Integrals, Series, and Products, 6th ed. San Diego, CA: Academic Press, 2000.

[19] Z. Wang, G.B. Giannakis, A simple and general parameterization quantifying performance in fading channels, IEEE Trans. Commun., 2003, 51(8): 1389-1398.

[20] K. Cho, D. Yoon, On the general BER expression of one and two dimensional amplitude modulation, IEEE Trans. Commun., 2002, 50(7): 1074-1080. 